\title{
A abordagem psicossomática na medicina geral e familiar: estudo transversal
}

Catarina Rebelo-Neves, ${ }^{1}$ Joana Nogueira, ${ }^{2}$ Carlos Amaral Dias, ${ }^{3}$ Jorge Torgal ${ }^{4}$

\section{RESUMO}

Objetivo: Caracterizar a forma como os Médicos de Família Portugueses percebem e integram a perspetiva psicossomática na sua prática clínica.

Tipo de estudo: Estudo transversal.

Local/População: Médicos especialistas em Medicina Geral e Familiar a exercer em Portugal (amostra de conveniência).

Método: Aplicou-se um questionário de autopreenchimento, entre agosto e novembro de 2013.

Resultados: Foram incluídos 100 médicos ( $77 \%$ do sexo feminino), com idades entre os 26 e os 70 anos. Os sujeitos fazem por integrar a perspetiva psicossomática na sua prática clínica e concordam com a definição de psicossomática apresentada.

Conclusões: Encontrámos indicadores no sentido da pertinência e necessidade de maior informação e orientação dos médicos de medicina geral e familiar.

Palavras-chave: Psicossomática; Medicina geral e familiar; Sintomas; Psicossocial

\section{INTRODUÇÃO}

ncontram-se, em todas as áreas do sistema de
cuidados de saúde, pacientes que apresentam
sintomas físicos sem uma causa orgânica
óbvia. ${ }^{1}$

A somatização é uma das problemáticas mais comuns nos serviços de saúde primários, estando associada a uma incapacidade funcional e elevado recurso a cuidados de saúde..$^{2-4}$ A categorização de doentes com sintomas sem explicação médica por parte dos médicos em cuidados primários revela variações substanciais. ${ }^{5}$

A apresentação persistente de sintomas sem explicação médica é um enorme desafio para os médicos com a especialidade de medicina geral e familiar (MGF), responsável por uma grande pressão na relação médico-doente. ${ }^{1,6}$ A presença destes sintomas oscila

1. Doutoramento em Ciências da Vida. Faculdade de Ciências Médicas da Universidade Nova de Lisboa.

2. Mestre em Psicologia Clínica. ISPA-Instituto Universitário.

3. Instituto Superior Miguel Torga.

4. Departamento de Saúde Pública. Faculdade de Ciências Médicas da Universidade Nova de Lisboa. desde situações que são leves e transitórias até manifestações múltiplas e severas. ${ }^{1,7-8}$

Os sintomas de somatização mais frequentemente apresentados em cuidados primários são: dor no peito, fadiga, tonturas, dores de cabeça, inchaço, dores nas costas, falta de ar, insónia, dormência e dor abdominal. ${ }^{8-9}$

Os doentes psicossomáticos investem numa procura de doença orgânica para combater o seu medo de a ter, apesar de não a terem. ${ }^{10-11}$ Neste sentido realizam-se, assim, inúmeras análises clínicas desnecessárias e dispendiosas, além de se poder desenvolver uma adição a substâncias ${ }^{12-14} \mathrm{e}$ de se deteriorar a relação médico-doente, por este último sentir que os fatores psicossociais são ignorados. ${ }^{10}$

Estudos demonstram que a prevalência de doentes em unidades de cuidados primários com sintomas sem explicação médica oscila entre $16 \%$ e $50 \%,{ }^{1,4,7-8,12,15}$ tornando-se estes sintomas a categoria mais comum de queixas em cuidados primários. ${ }^{2,16-17} \mathrm{O}$ facto de uma grande parte destes pacientes não preencher todos os critérios de referenciação para os serviços de saúde 
mental ${ }^{6}$ faz com que o tratamento dos mesmos recaia sobre os especialistas em MGF.

Os médicos com a especialidade de MGF reconhecem, maioritariamente, a importância de uma explicação adequada na apresentação do diagnóstico de sintomas sem explicação médica, embora se sintam muitas vezes incapazes de o fazer claramente aos seus doentes, tentando usar metáforas, normalizando os sintomas e explicando que não há doença física. ${ }^{18-20}$ Outros há que não reconhecem o valor que o seu coping e apoio têm no alívio dos sintomas e na satisfação destes doentes. ${ }^{21}$ Quando o doente persiste na vinda à consulta, os médicos optam muitas vezes por salvaguardar uma boa relação médico-doente e manter a rotina de visitas. ${ }^{11}$

Enquanto alguns médicos advogam uma maior precisão na identificação destes sintomas ${ }^{22}$ outros acreditam que as classificações específicas são insuficientes pois não combinam com a «nebulosidade» das doenças físicas e psicológicas apresentadas pelos doentes em cuidados primários. ${ }^{1,12,23}$

Pacientes com sintomas físicos sem explicação médica recusam muitas vezes a necessidade de apoio psicológico quando consultam um especialista em MGF, dando preferência a uma intervenção médica sobre os sintomas mas, ao mesmo tempo e indiretamente, demonstram o desejo de apoio emocional. ${ }^{24}$

Habitualmente, os tratamentos de sintomas sem explicação médica em cuidados primários de saúde são infrequentes e conduzem a resultados diversos. ${ }^{25}$ Os mesmos autores verificaram, em casos de alta satisfação com a relação médico-doente, efeitos de melhoria, moderados a elevados, no funcionamento mental e nas somatizações. A terapia cognitivo-comportamental tem-se revelado das mais eficazes na redução e extinção de sintomas sem explicação médica, reduzindo a sintomatologia física, o sofrimento psicológico, a incapacidade e o número de visitas à consulta, ${ }^{26-29}$ embora, em alguns casos, não se verifique maior eficácia do que a da consulta médica geral. ${ }^{?}$

Um estudo multidimensional ${ }^{28}$ revelou a eficácia de diretrizes focadas no uso de antidepressivos, na abordagem cognitivo-comportamental e na não utilização controlada de substâncias, ou seja, medicação para alívio dos sintomas físicos. Os pacientes revelaram melhorias na satisfação, redução da incapacidade física e dos sintomas depressivos. Os pacientes que adotaram melhores estratégias de coping apresentaram resultados mais favoráveis do que os que apenas se centraram em eliminar os sintomas físicos. ${ }^{30}$

O objetivo geral deste estudo é analisar o modo como os especialistas em MGF percecionam e integram a perspetiva psicossomática na sua prática clínica, mais precisamente no que respeita ao diagnóstico, à referenciação e ao encaminhamento de doentes com manifestações consideradas de índole psicossomática.

Um dos objetivos específicos deste estudo é investigar em que medida os respondentes integram a perspetiva do texto na sua prática clínica e, em caso afirmativo, obter um exemplo prático dessa aplicação.

No caso dos respondentes que indiquem não aplicar esta perspetiva, o questionário propõe que indiquem o seu grau de concordância.

\section{MÉTODO}

Tipo de estudo

O presente trabalho de investigação inscreve-se num estudo exploratório descritivo transversal. Abrange apenas um momento de avaliação, com uma amostra de médicos, da população portuguesa, com a especialidade de MGF.

\section{Material}

Recolha de dados a partir de um questionário para caracterização sociodemográfica: idade, género e anos de exercício da prática clínica. Questão principal sobre o grau de concordância relativa a um texto que contém uma definição de psicossomática. O grau de concordância relativo à definição apresentada é expresso numa escala de «Concordo»; «Concordo mais do que discordo»; «Discordo mais do que concordo»; e «Discordo». A definição de psicossomática apresentada foi:

"A Medicina Psicossomática pode ser definida como um quadro abrangente e interdisciplinar que permite: a avaliação de fatores psicológicos que afetam a vulnerabilidade individual bem como o curso e resultado da doença; a consideração biopsicossocial no atendimento ao doente na prática clínica; intervenções de especialistas para integrarem terapias psicológicas na prevenção, tratamento e reabilitação da doença médica. A Medicina Psicossomática é, por definição, multidisciplinar. Neste sentido, não se limita à área da Psiquiatria, antes podendo incidir sobre qualquer outro 
campo da Medicina. Curiosamente, a abordagem geral de Psicossomática resultou num número de subdisciplinas consoante as áreas de aplicação, entre as quais: Psico-oncologia, Psiconeurologia, Psiconeuroendocrinologia, Psicoimunologia, Psicodermatologia, entre outras. Os avanços atuais nesta área têm implicações para a pesquisa e para a prática médica, com particular incidência no papel do estilo de vida, no desafio dos sintomas sem explicação médica, nas necessidades psicossociais decorrentes da doença crónica, na avaliação da terapia além do reducionismo farmacêutico, na função de o paciente contribuir ativamente para a sua saúde. A área da Medicina Psicossomática tem-se revelado cada vez mais cientificamente rigorosa, diversificada e terapeuticamente relevante."

Fava GA, Sonino N. Psychosomatic medicine. Int J Clin Practice. 2010;64(10):1155-61.

O questionário introduziu ainda uma questão sobre a integração que os sujeitos fazem da mesma perspetiva na sua prática clínica e, se a resposta foi afirmativa, com acesso a uma pergunta aberta para descreverem e/ou darem exemplo de como o fazem ou de como gostariam de o poder fazer.

\section{Procedimento}

O instrumento utilizado no presente estudo, dirigido a médicos de MGF da população portuguesa, chegou até aos sujeitos por correio eletrónico, apresentando uma breve explicação sobre os objetivos e indicações de preenchimento.

\section{Aspetos éticos}

Foi garantido aos participantes o anonimato e confidencialidade dos dados fornecidos.

\section{Participantes}

Na elaboração deste estudo foram aplicados 113 questionários online, usando a plataforma Survey Monkey, no sentido de obter uma análise descritiva e aferir o diagnóstico e encaminhamento que são feitos a estes doentes nas consultas da especialidade de MGF.

\section{Amostra}

A amostra, de conveniência e não probabilística, ${ }^{31}$ foi recolhida no período entre agosto e novembro de 2013.
O número de participantes foi definido ad-hoc, tendo como referência o facto de estarmos perante um estudo exploratório e de que "amostras de 80 ou mesmo 40 indivíduos podem fornecer características sensíveis da população" ${ }^{32}$

Foram excluídos todos os participantes que desistiram e/ou não preencheram de modo completo o questionário até ao final. Com este critério de exclusão obteve-se uma amostra constituída por 100 médicos de MGF, sendo 23 do sexo masculino e 77 do sexo feminino. A idade dos sujeitos varia entre os 26 e os 70 anos. Os indivíduos exercem a atividade de MGF num intervalo variável (Quadro I).

\section{RESULTADOS}

Análise de dados

$\mathrm{Na}$ análise do grau de concordância com o texto apresentado, para um intervalo de confiança de $95 \%$ a maioria dos respondentes referiu concordar (66\%) ou concordar mais do que discordar (30\%).

Em 94\% dos casos a resposta quanto à aplicação desta perspetiva na prática clínica foi afirmativa, sendo negativa nos restantes 6\% (Figura 2). Cinco destes respondentes não fizeram comentários adicionais, não sendo possível, a partir dos dados recolhidos, inferir a razão de não integrarem a perspetiva apresentada.

Apenas um respondente, o qual respondeu «Concordar», referiu que considera que a área abordada pelo texto é desconhecida e que a sua divulgação é importante.

Inversamente existem três casos em que os respondentes referem «Discordar» ou «Discordar mais do que concordar»e, simultaneamente, indicam que integram a perspetiva na sua prática.

Dois destes sujeitos indicaram exemplos e um comentário, cuja transcrição se apresenta:

a) «Discorda mais do que concorda»

Exemplo: "As pessoas que me consultam são vistas como um todo. A abordagem biopsicossocial faz parte do meu método clínico e as características da minha prática que acentuam fortemente na relação médico-utente/doente/paciente dirigem-se a todos os problemas de saúde, independentemente da sua natureza, numa perspetiva integradora e coordenada de cuidados. A abordagem psicossomática é uma constante do meu trabalho clínico. Assim, todos os 
problemas são contextualizados, considerando o tipo de pessoa, a sua família, as suas relações sociais e de trabalho, as suas crenças e cultura, procurando sempre desenvolver a autonomia de quem me consulta." Comentário: "Os especialistas em MGF são aqueles que no dia-a-dia de trabalho mais trabalham esta área. Seria bom que este estudo tivesse em consideração o muito trabalho que nesta área tem sido feito nesta especialidade."

\section{b) «Discorda»}

Exemplo: "Através de avaliação de fatores biopsicossociais: deteção de fase de «crise» em qualquer um destes fatores. O acompanhamento continuado do doente é facilitador desta avaliação. Quando os sintomas/sinais decorrem de um período de "crise», e apoiado na personalidade individual, assim como em antecedentes pessoais, a causa-efeito pode ser evidente. O próprio utente, quando indagado e esclarecido, chegará simultaneamente à mesma conclusão, o que será positivo para medidas terapêuticas a adotar. Os métodos de avaliação familiar e individual, aplicados de forma informal fazem parte da avaliação global dos utentes."

Os casos em que o respondente refere "Concordar" ou «Concordar mais do que discordar» com o texto apresentado e que integra a perspetiva psicossomática na sua prática clínica perfazem 91.

Procedeu-se a uma análise de conteúdo dos exemplos para perceber como é que os respondentes integram a perspetiva psicossomática na sua prática clínica, tendo emergido desta análise de conteúdo três fatores principais [Escuta terapêutica/Abordagem Psicológica/Desencadeado por fatores emocionais (1); Abordagem psicossocial (2); Procura de outras abordagens/alternativas (3)] e uma categoria aberta, intitulada de Outros.

Tendo em conta os exemplos apresentados, parece haver uma tendência para o recurso a uma abordagem biopsicossocial e, noutros casos, a uma abordagem psicológica e de escuta terapêutica, reconhecendo que os sintomas podem ser desencadeados por fatores emocionais (Figura 2).

No que concerne à utilização da definição apresentada no questionário, entre os que responderam que a integram na sua prática clínica surgiram três categorias

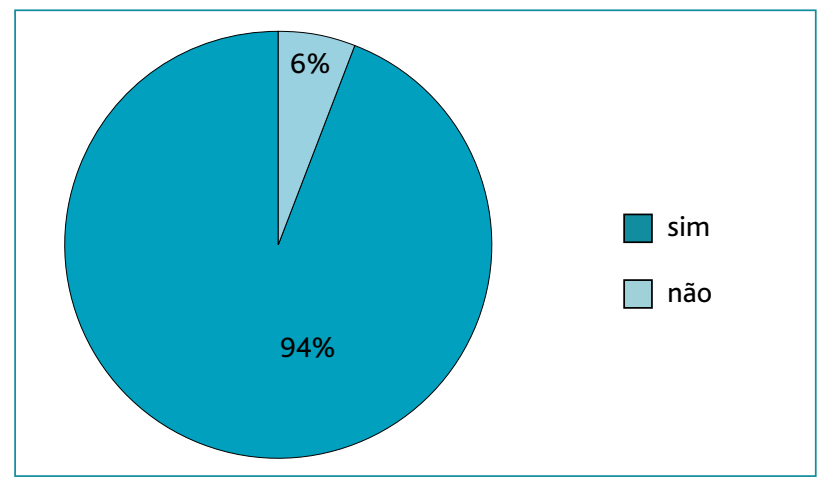

Figura 1. Integração da perspetiva psicossomática na prática clínica (\%).

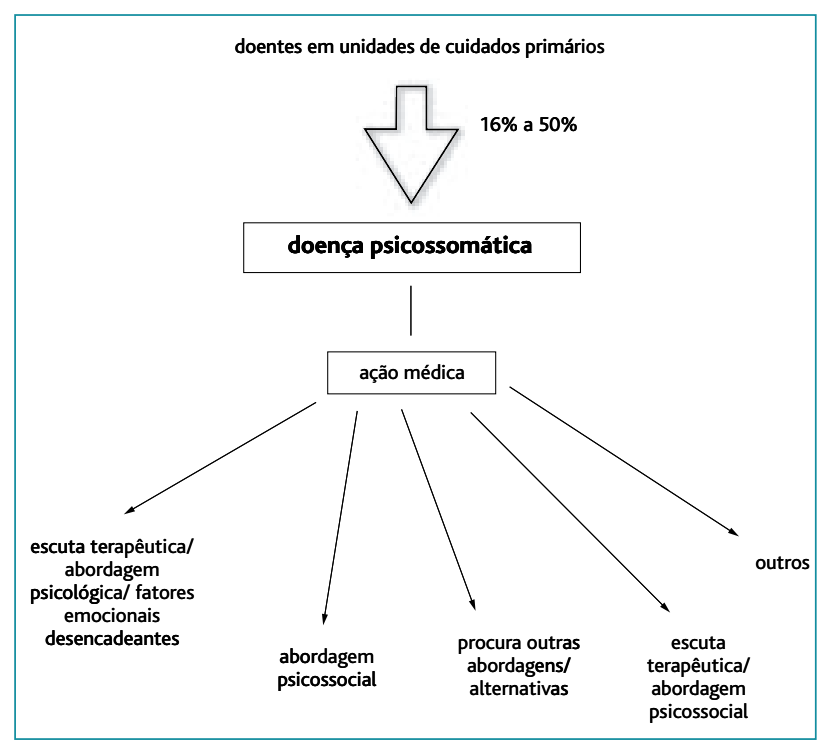

Figura 2. Categorias características da dispersão encontrada na prática clínica em cuidados de saúde primários.

principais: necessidade de acesso a equipa multidisciplinar; pouca informação científica que ajude a regular a sua prática neste âmbito; e dificuldades em adotar uma abordagem biopsicossocial. Nos resultados obtidos parece haver alguma unanimidade em relação às necessidades e às dificuldades sentidas pelos sujeitos. Apenas um dos respondentes, que não integra a perspetiva psicossomática na sua prática clínica, refere falta de informação nessa área.

\section{DISCUSSÃO}

A doença psicossomática tem uma incidência entre $16 \%$ e $50 \%$ entre os doentes em unidades de cuidados 
primários, , ${ }^{1,47-8,810,12}$ sendo considerada a categoria mais comum de queixas perante os médicos em cuidados primários. ${ }^{2,16-17,26}$

Neste sentido torna-se pertinente inquirir os médicos de cuidados de saúde primários se estes se consideram aptos a lidar com a perturbação e, mais do que isso, se na sua prática clínica integram uma abordagem adequada às necessidades das pessoas que apresentem manifestações psicossomáticas.

De forma a compreender se os médicos de MGF concordam e integram na sua prática clínica a noção de doença psicossomática foram levantadas algumas questões que, após uma análise dos resultados, nos permitiram refletir e discutir os resultados obtidos, à luz da literatura já existente nesta temática.

A abordagem dos médicos de MGF a pacientes com sintomas sem explicação médica varia de consulta para consulta, passando os doentes, previamente, por uma série de consultas focadas em aspetos físicos do problema antes de o médico levantar a possibilidade de os sintomas serem inexplicáveis medicamente." As perspetivas podem variar desde uma abordagem biomédica até uma abordagem biopsicossocial, mas os médicos em cuidados primários começam sempre por excluir as doenças físicas mais óbvias antes de procurarem por outras explicações para os sintomas. ${ }^{33}$ Já Enge $^{34}$ referia que era importante mostrar aos médicos com a especialidade de MGF que existe mais medicina para além da biomedicina. Nos anos seguintes acentuou-se esta preocupação e o cuidado com a abrangência das funções profissionais dos especialistas em MGF, culminando em 2002 com a declaração da WONCA, desenvolvida pelo grupo de trabalho no âmbito da European Society of General Practice/Family Medicine. Nasceu, assim, em 2005, um documento curto de apoio aos especialistas em MGF que define, de um modo muito mais abrangente que outrora, o âmbito desta disciplina. É acentuada, então, a responsabilidade quer pelo paciente individual quer pela comunidade mais alargada no que respeita às questões de saúde. A abordagem é holística, considerando as dimensões cultural e existencial integradas com a capacidade de usar um modelo biopsicossocial. ${ }^{35}$

Nesta linha, o presente estudo realçou a diversidade que existe na abordagem dos médicos de cuidados de saúde primários perante sintomas sem explicação médica, tendo-se destacado as seguintes categorias: Escu- ta terapêutica/Abordagem Psicológica/Desencadeado por fatores emocionais; abordagem psicossocial; Procura de outras abordagens/alternativas (Figura 2) ${ }^{\dagger}$

Hansen e colaboradores ${ }^{33}$ analisaram a abordagem dos médicos de MGF na consulta com doentes que manifestavam sintomas sem explicação médica, tendo obtido três tipos diferentes de consulta - procura por doença; rotina; várias alternativas -, sendo que os mesmos variaram, desde uma abordagem biomédica a uma abordagem onde múltiplas explicações foram levantadas para explicarem o problema do doente. A escolha do tipo de consulta foi influenciada pelo médico, pelo paciente e por fatores contextuais que afetavam o processo de diagnóstico. Estas observações coincidem com resultados em estudos de entrevista a doentes com sintomas sem explicação médica, em que estes relatam encontrar vários tipos de explicação quando consultam médicos diferentes. ${ }^{36}$

É notório que a investigação empírica em torno do acompanhamento de doentes com sintomas sem explicação médica, por parte dos especialistas em MGF, é muito escassa e dispersa.

Dependendo da abordagem que o médico escolhe, será dada maior ou menor relevância a aspetos psicossociais. De acordo com Ring e colaboradores, ${ }^{18}$ os médicos com a especialidade de MGF raramente envolvem sinais psicossociais no seu processo de diagnóstico.

Os médicos com a especialidade de MGF tendem a reconhecer a importância de uma explicação adequada no diagnóstico de sintomas sem explicação médica; no entanto, sentem-se muitas vezes incapazes de a apresentar claramente aos seus doentes. ${ }^{36}$ Há lacunas na formação a este nível, dado que a maioria dos especialistas dizem integrar uma perspetiva psicossomática na sua prática clínica, embora não seja o que depois se verifica nos exemplos dados. ${ }^{4}$

Os resultados obtidos revelaram que a maioria dos sujeitos concorda com a definição dada de psicossomática. No entanto, ainda há um grande caminho a percorrer quer na informação quer na orientação feita junto dos médicos de MGF. Apesar disso, é notória a

* Indicador no sentido da pertinência de um incremento de formação e informação sobre a abordagem psicossomática na prática clínica junto dos especialistas em MGF.

† Houve também uma categoria que conjugava a Escuta terapêutica e a abordagem biopsicossocial e uma categoria aberta Outras. 
preocupação e vontade de ter mais recursos e mais informação para lidar com este tipo perturbações.

Limitações: A amostra não permite generalizar os resultados a toda a população.

Teria sido interessante alargar as questões de investigação, por exemplo, solicitar aos sujeitos uma projeção de quantos doentes já acompanharam ou diagnosticaram com doença psicossomática e para que especialidade os encaminharam, bem como dos sintomas que parecem surgir predominantemente e a idade e género desses doentes.

Estudos futuros: Ao longo deste estudo foram surgindo diversas hipóteses para trabalhos futuros, que acreditamos que enriqueceriam a investigação em psicossomática nos cuidados primários. Assim, entendemos relevante fazer uma análise junto de outros profissionais de cuidados primários como, por exemplo, os enfermeiros e que se estude também esta temática numa abordagem centrada sobre a perspetiva dos doentes.

Muitos outros estudos prévios são ainda necessários para aferir da pertinência do desenvolvimento de um programa específico para os médicos com a especialidade de MGF, de cariz formativo e preventivo, sobre abordagem das doenças psicossomáticas, destinado quer aos especialistas em exercício quer aos que estão a receber formação ao nível da especialidade.

\section{CONCLUSÃO}

Os sujeitos desta amostra concordam com a definição de psicossomática apresentada e fazem por integrar a perspetiva psicossomática na sua prática clínica. Encontrámos indicadores no sentido da pertinência de um incremento de formação e informação sobre a abordagem psicossomática na prática clínica junto dos especialistas em MGF.

\section{REFERÊNCIAS BIBLIOGRÁFICAS}

1. Rosendal M, Olesen F, Fink P. Management of medically unexplained symptoms. BMJ. 2004;330(7481):4-5.

2. Burton $\mathrm{C}$. Beyond somatisation: a review of the understanding and treatment of medically unexplained physical symptoms (MUPS). $\mathrm{Br} J$ Gen Pract. 2003;53(488):231-9.

3. Fink P, Rosendal M, Toft T. Assessment and treatment of functional disorders in general practice: the extended reattribution and management model: an advanced educational program for nonpsychiatric doctors. Psychosomatics. 2002;43(2):93-131.

4. Kocalevent RD, Hinz A, Brähler E. Standardization of a screening ins- trument (PHQ-15) for somatization syndromes in the general population. BMC Psychiatry. 2013;13:91.

5. Rosendal M, Bro F, Fink P, Christensen KS, Olesen F. Diagnosis and somatization: effect of an educational intervention in a cluster randomized controlled trial. Br J Gen Pract. 2003;53(497):917-22.

6. Olde Hartman TC, Hassink-Franke LJ, Lucassen PL, van Spaendonck KP, van Weel C. Explanation and relations. How do general practitioners deal with patients with persistent medically unexplained symptoms: focus group study. BMC Fam Pract. 2009;10:68.

7. Arnold IA, de Waal MW, Eekhof JA, Assendelft WJ, Spinhoven P, van Hemert AM. Medically unexplained physical symptoms in primary care: a controlled study on the effectiveness of cognitive-behavioral treatment by the family physician. Psychosomatics. 2009;50(5):515-24.

8. Edwards TM, Stern A, Clarke DD, Ivbijaro G, Kasney LM. The treatment of patients with medically unexplained symptoms in primary care: a review of the literature. Ment Health Fam Med. 2010;7(4):209-21.

9. Hanel G, Henningsen P, Herzog W, Sauer N, Schaefert R, Szecsenyi J, et al. Depression, anxiety, and somatoform disorders: vague or distinct categories in primary care? Results from a large cross-sectional study. J Psychosom Res. 2009;67(3):189-97.

10. Luo Z, Goddeeris J, Gardiner JC, Smith RC. Costs of an intervention for primary care patients with medically unexplained symptoms: a randomized controlled trial. Psychiatr Serv. 2007;58(8):1079-86.

11. Nunes J, Ventura T, Encarnação R, Pinto PR, Santos I. What do patients with medically unexplained physical symptoms (MUPS) think? A qualitative study. Ment Health Fam Med. 2013;10(2):67-79.

12. Dowrick C, Gask L, Hughes JG, Charles-Jones H, Hogg JA, Peters S, et al. General practitioners' views on reattribution for patients with medically unexplained symptoms: a questionnaire and qualitative study. BMC Fam Pract. 2008;9:46.

13. Fink P. Surgery and medical treatment in persistent somatizing patients. J Psychosom Res. 1992;36(5):439-47.

14. Lightfoot RW Jr, Luft BJ, Rahn DW, Steere AC, Sigal LH, Zoschke DC, et al. Empiric parenteral antibiotic treatment of patients with fibromyalgia and fatigue and a positive serologic result for Lyme disease: a costeffectiveness analysis. Ann Intern Med. 1993;119(6):503-9.

15. Steinbrecher N, Koerber S, Frieser D, Hiller W. The prevalence of medically unexplained symptoms in primary care. Psychosomatics. 2011;52(3):263-71.

16. Katon W, Von Korff M, Lin E, Simon G, Walker E, Unützer J, et al. Stepped collaborative care for primary care patients with persistent symptoms of depression: a randomized trial.Arch Gen Psychiatry. 1999;56(12):1109-15.

17. Kirmayer LJ, Tailefer S. Somatoform disorders. In: Turner SM, Hersen M, editors. Adult psychopathology and diagnosis. 3rd ed. New York:Wiley; 1997. p. 333-83.

18. Ring A, Dorwick C, Humphris G, Salmon P. Do patients with unexplained physical symptoms pressurize general practitioners for somatic treatment? A qualitative study. BMJ. 2004;328(7447):1057-60.

19. Salmon P, Dowrick CF, Ring A, Humphris GM. Voiced but unheard agendas: qualitative analysis of the psychosocial cues that patients with unexplained symptoms present to general practitioners. $\mathrm{Br} J$ Gen Pract. 2004;54(500):171-6.

20. Salmon P, Ring A, Dowrick CF, Humphris GM. What do general practice patients want when they present medically unexplained symptoms, 
and why do their doctors feel pressurized? J Psychosom Res. 2005;59(4): 255-60.

21. Nordin TA, Hartz AJ, Noyes R Jr, Anderson MC, Rosenbaum ME, James $\mathrm{PA}$, et al. Empirically identified goals for the management of unexplained symptoms. Fam Med. 2006;38(7):476-82.

22. Brown RJ. Introduction to the special issue on medically unexplained symptoms: background and future directions. Clin Psychol Rev. 2007;27(7):769-80.

23. Rosendal M, Fink P, Falkoe E, Hansen HS, Olesen F. Improving the classification of medically unexplained symptoms in primary care. Eur J Psychiat. 2007;21(1):25-36.

24. Salmon P, Ring A, Humphris GM, Davies JC, Dowrick CF. Primary care consultations about medically unexplained symptoms: how do patients indicates what they want? J Gen Intern Med. 2009;24(4):450-6.

25. Smith RC, Gardiner JC, Luo Z, Schooley S, Lamerato L, Rost K. Primary care physicians treat somatization. J Gen Intern Med. 2009;24(7):82932.

26. Kroenke K. Efficacy of treatment for somatoform disorder: a review of randomized controlled trial. Psychosom Med. 2007;69(9):881-8.

27. Martin A, Rauh E, Fichter M, Rief W. A one-session treatment for patients suffering from medically unexplained symptoms in primary care: a randomized clinical trial. Psychosomatics. 2007;48(4):294-303.

28. Smith RC, Lyles JS, Gardiner JC, Sirbu C, Hodges A, Collins C, et al. Primary care clinicians treat patients with medically unexplained symptoms: a randomized controlled trial. J Gen Intern Med. 2006;21(7):671-7.

29. Sumathipala A. What is the evidence for the efficacy of treatments for somatoform disorders? A critical review of previous intervention studies. Psychosom Med. 2007;69(9):889-900.

30. Barsky AJ, Ahern DK. Cognitive behavior therapy for hypochondriasis: a randomized controlled trial. JAMA. 2004;291(12):1464-70.
31. Marôco J. Análise estatística com o PASW Statistic. Pêro Pinheiro: Report Number; 2010.

32. Pais-Ribeiro JL. Metodologia de investigação em psicologia e saúde. $2^{\mathrm{a}}$ ed. Porto: Legis Editora; 2008. ISBN 9789898148162

33. Hansen HS, Rosendal M, Fink P, Risor MB. The general practitioner's consultation approaches to medically unexplained symptoms: a qualitative study. ISRN Fam Med. 2013;2013:ID541604.

34. Engel GL. The need for a new medical model: a challenge for biomedicine. Science. 1977;196(4286):129-36.

35. Allen J, Gay B, Crebolder H, Heyrman J, Svab I, Ram P, et al. A definição europeia de medicina geral e familiar (clínica geral/medicina familiar) [The European definition of family medicine (general practice/family medicine)]. Rev Port Clin Geral. 2005;21(5):511-6. Portuguese

36. Salmon P. Conflict, collusion or collaboration in consultations about medically unexplained symptoms: the need for a curriculum of medical explanation. Patient Educ Couns. 2007;67(3):246-54.

\section{CONFLITO DE INTERESSES}

Os autores declaram não possuir quaisquer conflitos de interesse.

\section{FINANCIAMENTO}

Este trabalho foi elaborado sem qualquer tipo de financiamento.

\section{ENDEREÇO PARA CORRESPONDÊNCIA}

Catarina Rebelo-Neves

Edifício Hollywood, R. Luís Augusto Palmeirim, 19-1 A, 1700-273 Lisboa

E-mail: crebeloneves@gmail.com

Recebido em 06-11-2016

Aceite para publicação em 12-03-2018

\section{ABSTRACT}

\section{THE PSYCHOSOMATIC APPROACH IN PORTUGUESE GENERAL AND FAMILY MEDICINE: A CROSS-SECTIONAL STUDY}

Aim: To establish how the Portuguese general and family specialists perceive and integrate the psychosomatic perspective in their practice.

Design: Cross-sectional study.

Setting: Portuguese general and family practitioners.

Method: Providers answered a self-reported survey from August to November of 2013. Self-reported data collected from 100 Portuguese general and family medicine doctors. Participants are between 26 and 70 years old, $77 \%$ female and $23 \%$ male. Results: A hundred doctors participated in the survey (77\% females) with age from 26 to 77 years old. Professionals use the psychosomatic approach in their clinical practice and they agree with the definition of psychosomatic provided.

Conclusions: We find indicators towards the relevance and need for greater information and guidance of general practice physicians.

Keywords: Psychosomatic; General and family medicine; Symptoms; Psychosocial 